\title{
SHAPLEY-SHUBIK AND BANZHAF INDICES REVISITED
}

\section{Annick Laruelle and Federico Valenciano}

\author{
WP-AD 2000-02
}

Correspondence to A. Laruelle: Universidad de Alicante. Departamento de Fundamentos del Análisis Económico. Campus San Vicente. 03071 Alicante, Spain.

Editor: Instituto Valenciano de Investigaciones Económicas, s.a.

First Edition January 2000.

Depósito Legal: V-114-2000

IVIE working papers offer in advance the results of economic research under way in order to encourage a discussion process before sending them to scientific journals for their final publication.

* We want to thank M. Maschler and J. M. Zarzuelo for their insightful comments. This research has been supported by the Training and Mobility of Researchers programme initiated by the European Commission, by the Instituto Valenciano de Investigaciones Económicas (IVIE), and by the DGES of the Spanish Ministerio de Educación y Cultura, under project PB96-0247.

** A. Laruelle: Universidad de Alicante; F. Valenciano: Universidad del País Vasco. 


\title{
SHAPLEY-SHUBIK AND BANZHAF INDICES REVISITED
}

\author{
Annick Laruelle and Federico Valenciano
}

\section{A B S T R A C T}

We provide a new axiomatization of the Shapley-Shubik and the Banzhaf power indices in the domain of simple superadditive games by means of transparent axioms. Only anonymity is shared with the former characterizations in the literature. The rest of the axioms are substituted by more transparent ones in terms of power in collective decisionmaking procedures. In particular, a clear restatement and a compelling alternative for the transfer axiom are proposed. Only one axiom differentiates the characterization of either index, and these differentiating axioms provide a new point of comparison. In a first step both indices are characterized up to a zero and a unit of scale. Then both indices are singled out by simple normalizing axioms.

Key words: Power indices, voting power, collective decision-making, simple games, axiomatization. 


\section{Introduction}

Shapley-Shubik (1954) proposed the specialization of the Shapley (1953) value to assess the a priori measure of power of each agent in a collective decision-making procedure modelled as a simple game. Assuming their interpretation of power as the chance to be critical for the passage of a decision, Banzhaf $(1965,1966)$ criticized the weights that the Shapley-Shubik index gives to coalitions of different sizes and proposed his own index. These indices had no axiomatic foundation till Dubey (1975) and Dubey and Shapley (1979) axiomatically characterized them on the class of simple games. Since then, several axiomatizations of both indices have been proposed (Owen (1978, 1982), Bolger (1982), Lehrer (1988), Haller (1994), Feltkamp (1995), Nowak (1997), Albizuri and Ruiz (1999) and Khmelnitskaya (1999)). The main motivations of these papers are either the mathematical challenge of finding a self-contained characterization in subclasses of TU-games (like simple games or simple superadditive games) or the lack of intuitive appeal of some axioms. In the process new indices have even been proposed (Deegan and Packel (1978), Johnston (1978), Holler and Packel (1983)). However it can be said that so far no power index is characterized by means of an intuitively compelling set of axioms (Felsenthal and Machover (1995)). Moreover, the axiomatic approach by itself is insufficient to settle the question of the choice of a power index (Laruelle (1999)).

The aim of this paper is to provide an axiomatization of the Shapley-Shubik and the Banzhaf indices as measures of power in collective decision-making procedures. Consequently our domain is the class of simple superadditive games. Indeed, the basic information specifying a collective decision-making procedure, that is, which coalitions can make a decision and which cannot, can be represented by a (0-1)-game, while monotonicity and superadditivity are natural requirements that guarantee some consistency of the procedure. With this aim in mind, we look for a set of clear and transparent axioms, that is, assumptions that, whatever their plausibility, have a clear meaning and make sense one by one, independently of the others. In particular, no importance is attached to the one/zero values of the characteristic function of a simple game describing a decision rule beyond the yes/no information they embody.

Our starting point and basic reference are the axiomatizations proposed by Dubey (1975) and Dubey and Shapley (1979), who characterize both indices by a set of four axioms. Three of them ("anonymity", "null player" and "transfer") are common to both indices, while the fourth one ("efficiency" and the below-called "Banzhaf total power") distinguishes both indices. If mathematically very elegant, these characterizations are not completely satisfactory in the context of simple superadditive games as models of decision-making procedures. Indeed, the meaning of the "transfer" axiom is a bit obscure. 
Similarly the differentiating axioms for the Shapley-Shubik index and the Banzhaf index lack of appeal in the context of collective decision-making procedures. On the one hand, as often criticized, the "Banzhaf total power" axiom has some unavoidable ad hoc flavor: the index it helps to characterize is partially within it. On the other hand, the corresponding axiom for the Shapley-Shubik index, "efficiency", obvious in other contexts and accepted in this one by sheer habit, is no more compelling in this context (see Laruelle and Valenciano (1999)).

In our system only "anonymity", which has a clear and compelling meaning, is preserved untouched. The rest of the axioms are substituted by more transparent ones. In particular our axioms deliberately exclude in a first step any normalizing ingredient. This allows us to characterize either index up to the choice of a zero and a unit of scale. The traditional "null player" axiom is replaced by a more natural and clear assumption about null players: just that this is the worst role any player can play in a game. "Transfer" is reformulated in an equivalent but more transparent version stating that the effect on the power of deleting a minimal winning coalition from the set of winning ones is the same in any game in which this coalition is minimal winning. This kind of "modified game", the one resulting from a game by dropping just one minimal winning coalition, is crucial in our axiomatic system. Then we show that transfer can be replaced by a simpler and more compelling axiom just stating that the effect of dropping one minimal winning coalition is the same for all players inside (resp., outside) this coalition. Our differentiating axioms are two assumptions concerning again the effect of dropping just one minimal winning coalition in a game. Our substitute for "efficiency" states that when a minimal winning coalition is dropped, the total loss in terms of power of the players within this coalition is equal to the total gain of the members of the complementary coalition, while our substitute for "Banzhaf total power" states the same in terms of average (instead of total) loss and gain. By means of these axioms the Shapley-Shubik and Banzhaf indices are characterized up to an additive and a positive multiplicative constants. In other words, up to the choice of a "zero" and a "unit of scale" for the measure of power. Stripping our axioms in this way of any normalizing component helps to clarify the role and meaning of each of them. We also discuss the absolute/relative issue and show how adding two purely normalizing axioms, the Shapley-Shubik index and the Banzhaf index are singled out. Finally, we deal with the extension of the indices to make comparison of power in games with different numbers of players.

The paper is organized as follows: in Section 2 the basic game theoretical background together with Dubey's, and Dubey and Shapley's characterizations are briefly reviewed. In Section 3 we present our axioms and in Section 4 their relationships with the traditional 
ones are established. Section 5 contains the main result of this paper: the characterization of both power indices up to a multiplicative and an additive constants. In Section 6 the absolute/relative issue is discussed. Then by adding two normalizing axioms we single out both indices. Section 7 deals with the extension of the indices to compare the power of players in situations involving different numbers of players. Finally, Section 8 closes this paper with some remarks emphasizing the main conclusions of this work and a brief discussion on some lines for further research.

\section{Background}

We recall here the necessary definitions to model a collective decision-making procedure. A cooperative transferable utility (TU) game is a pair $(N, v)$, where $N=\{1, \ldots, n\}$ denotes the set of players and $v$ a function which assigns a real number to each non-empty subset or coalition of $N$, and $v(\emptyset)=0$. The number of players in a coalition $S$ is denoted $s$. A (0-1)-game is a game in which the function $v$ only takes the values 0 and 1 . It is a simple game if it is not identically 0 , and obeys the condition of monotonicity: $v(T) \geq v(S)$ whenever $T \supseteq S$. In these games a coalition $S$ is winning if $v(S)=1$, and is losing if $v(S)=0$. A winning coalition is minimal if it does not contain any other. A player $i$ is said to be a swinger in a coalition $S$ if $S$ is winning and $S \backslash\{i\}$ is not. A player $i$ is said to be a null player if she or he is not a swinger in any coalition, that is, if $v(S)=v(S \backslash\{i\})$ for all $S$. In a simple game $v, W(v)$ (resp., $M(v)$ ) will denote the set of winning (resp., minimal winning) coalitions, and $w(v)$ (resp., $m(v)$ ) its number. Any of these sets, $W(v)$ or $M(v)$, fully characterizes the game $v$. For any coalition $S \subseteq N$, the $S$-unanimity game, denoted $\left(N, u^{S}\right)$, is the simple game

$$
u^{S}(T)= \begin{cases}1 & \text { if } T \supseteq S \\ 0 & \text { otherwise. }\end{cases}
$$

A TU game is superadditive if $v(S \cup T) \geq v(S)+v(T)$ whenever $S \cap T=\emptyset$. In the context of simple games, the superadditivity property is equivalent to the condition: $v(S)+v(N \backslash S) \leq 1$ for all $S \subset N$. Let $S G_{n}$ denote the set of all simple superadditive $n$-person games. When $N$ is clear from the context we refer to game $(N, v)$ as game $v$.

As a collective decision-making procedure is specified by the voting body and the decision rules, it can be modelled by a (0-1)-game whose winning coalitions are those that can make a decision without the vote of the remaining players. We assume that the decision rules are consistent in the following sense. The unanimity of the players can make a decision. Any subgroup of a group of voters that cannot make a decision cannot either. Two nonintersecting groups of voters cannot make decision at the same time. Therefore, 
under these conditions, collective decision-making procedures can be modelled as simple superadditive games.

A power index is a function $\Phi: S G_{n} \rightarrow R^{n}$ that associates with each simple superadditive game $v$ a vector or power profile $\Phi(v)$ whose $i$ th component is interpreted as a measure of the influence that player $i$ can exert on the outcome. To evaluate the distribution of power among the players the two best known power indices are the Shapley-Shubik (1954) index and the Banzhaf (1965) index. For a game $v$, the Shapley-Shubik index is given by

$$
S h_{i}(v)=\sum_{\substack{S \subseteq N \\(S \ni i)}} \frac{(s-1) !(n-s) !}{n !}(v(S)-v(S \backslash\{i\})), i=1, \ldots, n .
$$

The Banzhaf index is given by

$$
B z_{i}(v)=\frac{1}{2^{n-1}} \sum_{\substack{S \subseteq N \\(S \ni i)}}(v(S)-v(S \backslash\{i\})), i=1, \ldots, n .
$$

The Shapley-Shubik index was first axiomatized by Dubey (1975). Dubey and Shapley (1979) proposed the first axiomatization of the Banzhaf index. Theorem 1 below contains their results for the domain of simple superadditive games.

Anonymity (An): For all $v \in S G_{n}$, any permutation $\pi$ of $N$, and any $i \in N$,

$$
\Phi_{i}(\pi v)=\Phi_{\pi(i)}(v)
$$

where $(\pi v)(S):=v(\pi(S))$.

Null Player (NP): For any $v \in S G_{n}$ and any $i \in N$, if $i$ is a null player in game $v$, then

$$
\Phi_{i}(v)=0
$$

Transfer (T): For any $v, w \in S G_{n}$ such that $v \vee w \in S G_{n}$,

$$
\Phi(v)+\Phi(w)=\Phi(v \wedge w)+\Phi(v \vee w)
$$

where $(v \wedge w)(S):=\min \{v(S), w(S)\}$ and $(v \vee w)(S):=\max \{v(S), w(S)\}$.

Shapley Total Power or "Efficiency" (ShTP): For all $v \in S G_{n}$,

$$
\sum_{i=1}^{n} \Phi_{i}(v)=1 .
$$


Banzhaf Total Power (BzTP): For all $v \in S G_{n}$,

$$
\sum_{i=1}^{n} \Phi_{i}(v)=\frac{1}{2^{n-1}} \sum_{i=1}^{n} \sum_{\substack{S \subseteq N \\ S \ni i}}(v(S)-v(S \backslash\{i\})) .
$$

Theorem 1 Let $\Phi: S G_{n} \rightarrow R^{n}$, then

(i) The only $\Phi$ that satisfies anonymity (An), null player (NP), transfer (T) and Shapley total power (ShTP) is the Shapley-Shubik index.

(ii) The only $\Phi$ that satisfies anonymity (An), null player (NP), transfer (T) and Banzhaf total power (BzTP) is the Banzhaf index.

\section{More transparent axioms}

Now we revise Dubey and Shapley's axioms. That is, their axioms are discussed one by one, and substitutes for almost all of them are proposed: only anonymity (An) will be kept unchanged. The meaning of this axiom is clear and compelling: the power of each player in a simple superadditive game does not depend on how the players are labelled.

With respect to the null player (NP) axiom it should be noted that, despite its apparent plausibility, this axiom isolated just states that the power of any null player in any game is the same. Postulating that this power is zero means more than just this only once one adds something else. For instance, that the power of any player in any game is greater or equal than zero. But this is none of Dubey and Shapley's axioms. In fact, in their system NP only yields its full meaning together with the other three axioms. So, we will use instead the following axiom, that we call "null player*" in order to avoid a confusing and unnecessary multiplicity of names ${ }^{1}$ :

Null Player* (NP*): For all $v \in S G_{n}$, and all $i \in N$,

$$
i \text { is a null player in } v \Leftrightarrow \text { for all } w \in S G_{n}, \Phi_{i}(v) \leq \Phi_{i}(w) .
$$

The axiom is clear and compelling, and makes full sense by itself without requiring the company of any other. It states that being a null player is the worst role any player can expect to play, the role that yields a minimal measure of power. Mind the equivalence postulated in the axiom: it excludes trivial flat measures of power, so, it really says that being a null player is strictly the worst.

The transfer ( $\mathrm{T}$ ) axiom plays in the context of simple games the same role that linearity plays for general transferable utility games. It has often been considered opaque (Roth

\footnotetext{
${ }^{1}$ We will do the same with our reformulation of transfer, and its abreviation.
} 
(1977), Straffin (1982)), and it is particularly obscure in the context of collective decisionmaking (Felsenthal and Machover (1995)). We will use an equivalent but more transparent formulation of this condition. For it and for our other axioms, we need the following definition that plays a central role in our axiomatization.

Definition 1 For any game $v \in S G_{n}$ such that $v \neq u^{N}$, and any $S \in M(v)$, the modified game $v_{S}^{*}$ is the game such that $W\left(v_{S}^{*}\right)=W(v) \backslash\{S\}$.

Thus the modified game $v_{S}^{*}$ results from $v$ by deleting just one single coalition $S$ from the list of winning ones ${ }^{2}$. Avoiding starting from the unanimity game and dropping a minimal winning coalition guarantee that $v_{S}^{*} \in S G_{n}$. It makes sense for the measurement of power to evaluate the effects of modifying a decision-making rule in such a way that a coalition that previously could make a decision cannot any more.

Now transfer can be reformulated like this:

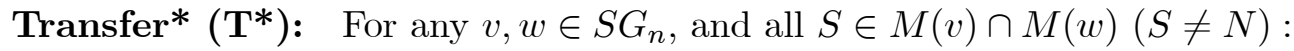

$$
\Phi_{i}(v)-\Phi_{i}\left(v_{S}^{*}\right)=\Phi_{i}(w)-\Phi_{i}\left(w_{S}^{*}\right)(\text { for all } i \in N) .
$$

This axiom postulates that the effect (gain or loss) on any player's power of eliminating a single minimal winning coalition from the set of winning ones is the same in any game in which this coalition is minimal winning. If equivalent to transfer as it will be proved later ${ }^{3}$, it seems less opaque. Note that this condition requires, for any $S$, a relation involving all games with $S$ as a minimal winning coalition and their modified forms. Instead we propose a condition that involves each game and its modified form. As we will show the following condition can replace transfer in our characterization.

Symmetric Gain-Loss (SymGL): For all $v \in S G_{n}$, all $S \in M(v)(S \neq N)$, and all $i, j \in S$ (resp., $i, j \in N \backslash S$ ),

$$
\Phi_{i}(v)-\Phi_{i}\left(v_{S}^{*}\right)=\Phi_{j}(v)-\Phi_{j}\left(v_{S}^{*}\right) .
$$

This assumption states that the effect of eliminating a minimal winning coalition is the same for any two players belonging to it and for any two players outside it. This axiom is a form of symmetry postulated for each pair $v$ and $v_{S}^{*}$. It is worth noting that this condition is satisfied by any semivalue (Weber $(1979,1988)$, see also Einy (1987)) and even by any weak semivalue (Calvo and Santos (1999)) in a weaker form.

\footnotetext{
${ }^{2}$ This is the "deletion" used by Weber (1988).

${ }^{3}$ In fact Dubey and Shapley observe that their axiom can be restated in terms of the effect of "changing the status of a single coalition from minimal winning to maximal losing".
} 
Now let us consider the differentiating axioms in Dubey and Shapley's axiomatization. What we have called "Shapley total power" is in fact the usual "efficiency" in the domain of TU games restricted to simple superadditive games. This condition is so compelling in the context of TU games that it is often uncritically accepted as natural in any subclass of TU games. But when simple superadditive games are interpreted as models of collective decision-making procedures and value as a measure of power, this condition lacks a compelling interpretation. In this case the $1 / 0$ values of the characteristic function is just a means to presenting the list of winning and losing coalitions. Thus, a priori, there is no reason to assume that there is any "cake" of a unit of power to distribute among the players. Moreover, the situation is similar to that of NP commented above: this "efficiency" isolated just states that the total aggregated power of the players is the same in any game.

If the lack of a compelling story for efficiency in this context, by sheer habit, may be not perceived at first sight, the corresponding axiom for the Banzhaf index, that we have called "Banzhaf total power", raises suspicion at first sight: the solution it helps to axiomatize is partly within the axiom.

We will use instead the following two axioms:

Constant Total Gain-Loss Balance (CTGLB): For all $v \in S G_{n}$ and all $S \in M(v)$ $(S \neq N)$,

$$
\sum_{i \in S}\left(\Phi_{i}(v)-\Phi_{i}\left(v_{S}^{*}\right)\right)=\sum_{j \in N \backslash S}\left(\Phi_{j}\left(v_{S}^{*}\right)-\Phi_{j}(v)\right) .
$$

Constant Average Gain-Loss Balance (CAGLB): For all $v \in S G_{n}$ and all $S \in$ $M(v)(S \neq N)$,

$$
\frac{1}{s} \sum_{i \in S}\left(\Phi_{i}(v)-\Phi_{i}\left(v_{S}^{*}\right)\right)=\frac{1}{n-s} \sum_{j \in N \backslash S}\left(\Phi_{j}\left(v_{S}^{*}\right)-\Phi_{j}(v)\right) .
$$

Both axioms again concern the effect of dropping a minimal winning coalition from the list of winning ones. Constant total (resp., average) gain-loss balance postulates that the total (resp., average) loss of the players in the deleted coalition equals the total (resp., average) gain of the players outside it. It is remarkable how close these two axioms are in their form and requirement: just one word -total/average- separates them. As to their appeal it seems quite balanced. In fact both conditions are very particular cases of the following principle involving the average gain and loss and satisfied by the semivalues:

$\lambda$-Average Gain-Loss Balance: For all $v \in S G_{n}$ and all $S \in M(v)(S \neq N)$,

$$
\lambda_{s} \frac{1}{s} \sum_{i \in S}\left(\Phi_{i}(v)-\Phi_{i}\left(v_{S}^{*}\right)\right)=\left(1-\lambda_{s}\right) \frac{1}{n-s} \sum_{j \in N \backslash S}\left(\Phi_{j}\left(v_{S}^{*}\right)-\Phi_{j}(v)\right),
$$

where $\lambda=\left(\lambda_{s}\right)_{s=1,2 . ., n-1}$, is a collection of constants $\lambda_{s} \in(0,1)$. 
It can be shown that each collection $\lambda$ is associated to a particular semivalue. Constant total gain-loss balance is the particular case $\lambda_{s}=\frac{s}{n}$, while constant average is the particular case $\lambda_{s}=\frac{1}{2}$.

These axioms turn out even close to our formulation of transfer $\left(\mathrm{T}^{*}\right)$ or its substitute (SymGL). All these axioms postulate something concerning the effect of eliminating one minimal winning coalition on the measurement of power, while the corresponding axioms in the other existing characterizations do not have anything in common. In Section 5 we discuss the meaning of this fact.

\section{Old and new axioms' relationships}

Before proceeding with the main results, we establish some relations between our axioms and Dubey and Shapley's that will be useful in the proof of the main theorems.

Note that our null player* is neither weaker nor stronger than traditional null player. The next proposition shows that our substitute together with anonymity implies that all null player roles are interchangeable or equivalent in the sense of yielding the same measure of power, the basic meaning of null player as commented before.

Proposition 1 Anonymity (An) together with null player* $\left(N P^{*}\right)$ implies that for all $i, j \in N$, and all $v, w \in S G_{n}$, if $i$ is a null player in $v$, and $j$ is a null player in $w$, then $\Phi_{i}(v)=\Phi_{j}(w)$.

Proof. Let $i$ be a null player in a game $v$, and let $w$ be any other game. Let $\pi$ be the permutation on $N$ interchanging $i$ and $j$, that is,

$$
\pi(k):=\left\{\begin{array}{l}
j, \text { if } k=i \\
i, \text { if } k=j \\
k \text { otherwise }
\end{array}\right.
$$

Then by NP* and An we have $\Phi_{i}(v) \leq \Phi_{i}(\pi w)=\Phi_{\pi(i)}(w)=\Phi_{j}(w)$, and the conclusion follows immediately.

For the sake of completeness we prove the equivalence of transfer and our reformulation of it.

Proposition 2 Transfer $(T)$ and transfer* $\left(T^{*}\right)$ are equivalent. 
Proof. $(\Rightarrow)$ Let $v, w \in S G_{n}$, and $S \in M(v) \cap M(w)(S \neq N)$. Then $v_{S}^{*}, w_{S}^{*} \in S G_{n}$ and $v=v_{S}^{*} \vee u^{S}$ and $w=w_{S}^{*} \vee u^{S}$. Then, by $\mathrm{T}$, we have

$$
\begin{aligned}
\Phi(v) & =\Phi\left(v_{S}^{*} \vee u^{S}\right)=\Phi\left(v_{S}^{*}\right)+\Phi\left(u^{S}\right)-\Phi\left(v_{S}^{*} \wedge u^{S}\right) \\
\Phi(w) & =\Phi\left(w_{S}^{*} \vee u^{S}\right)=\Phi\left(w_{S}^{*}\right)+\Phi\left(u^{S}\right)-\Phi\left(w_{S}^{*} \wedge u^{S}\right)
\end{aligned}
$$

Note that $v_{S}^{*} \wedge u^{S}=w_{S}^{*} \wedge u^{S}=\left(u^{S}\right)_{S}^{*}$, hence $\mathrm{T}^{*}$ follows immediately.

$(\Leftarrow)$ Conversely, let $v, w \in S G_{n}$ s. t. $v \vee w \in S G_{n}$. Note $W(v \wedge w)=W(v) \cap W(w)$ and $W(v \vee w)=W(v) \cup W(w)$. That is, $W(v \vee w) \backslash W(w)=W(v) \backslash W(v \wedge w)$. This means that reaching $w$ from $v \vee w$ takes dropping one by one exactly the same winning coalitions (a minimal one in the current game each time) than reaching $v \wedge w$ from $v$. By $\mathrm{T}^{*}$ the effect on the index of deleting a minimal wining coalition is the same in any game, consequently, $\Phi(v \vee w)-\Phi(w)=\Phi(v)-\Phi(v \wedge w)$.

As to our substitute for transfer, that is, symmetric gain-loss, it is independent of transfer. That is, it does not imply nor is implied by transfer. But the following proposition and next example show that our condition is weaker than transfer if anonymity is assumed.

Proposition 3 Anonymity (An) together with transfer* $\left(T^{*}\right)$ implies symmetric gain-loss (SymGL).

Proof. First observe that for any game $v$, any permutation $\pi$, and any $S \in M(\pi v)$, it is $(\pi v)_{S}^{*}=\pi\left(v_{\pi(S)}^{*}\right)$. This can easily be proved checking that both games have the same set of winning coalitions. Now let $v \in S G_{n}, S \in M(v)$, and $i, j \in S(i \neq j)$. Let $\pi$ be the permutation on $N$ interchanging $i$ and $j$, as in the proof of Proposition 1. Then $\pi(S)=S$, so that $S \in M(v) \cap M(\pi v)$. Then, by $\mathrm{T}^{*}$ and An (note that now $\left.(\pi v)_{S}^{*}=\pi\left(v_{\pi(S)}^{*}\right)=\pi\left(v_{S}^{*}\right)\right)$, we have

$$
\begin{aligned}
\Phi_{i}(v)-\Phi_{i}\left(v_{S}^{*}\right) & =\Phi_{i}(\pi v)-\Phi_{i}\left((\pi v)_{S}^{*}\right)=\Phi_{i}(\pi v)-\Phi_{i}\left(\pi\left(v_{S}^{*}\right)\right) \\
& =\Phi_{\pi(i)}(v)-\Phi_{\pi(i)}\left(v_{S}^{*}\right)=\Phi_{j}(v)-\Phi_{j}\left(v_{S}^{*}\right) .
\end{aligned}
$$

The proof for $i, j \in N \backslash S$ is entirely similar.

The following example shows that the converse is not true.

Example: Let $\Phi: S G_{3} \rightarrow R^{3}$ be the index that associates to each three person game the vector below (in the other cases just extend $\Phi$ anonymously):

If $M(v)=\{\{1\}\}$ then $\Phi(v)=(1,0,0)$;

if $M(v)=\{\{1,2\},\{1,3\},\{2,3\}\}$ then $\Phi(v)=(0.8,0.8,0.8)$;

if $M(v)=\{\{1,2\},\{1,3\}\}$ then $\Phi(v)=(0.9,0.3,0.3)$; 
if $M(v)=\{\{1,2\}\}$ then $\Phi(v)=(0.6,0.6,0)$;

if $M(v)=\{\{1,2,3\}\}$ then $\Phi(v)=(0.2,0.2,0.2)$.

Then $\Phi$ satisfies AN and SymGL. But, for each two person coalition, $\Phi_{i}(v)-\Phi_{i}\left(v_{S}^{*}\right)$, depending on the game, takes the values $0.5,0.3$ and 0.4 . Thus $\Phi$ does not satisfy $\mathrm{T}^{*}$.

The following proposition shows that "constant total power" would be perfectly justified as an alternative name for constant total gain-loss balance. In other words, this axiom captures exactly the essential meaning of the Shapley total power or "efficiency" axiom commented above: the sum of the power of all the players in any game is the same.

Proposition 4 Constant total gain-loss balance (CTGLB) is equivalent to requiring that for all $v, w \in S G_{n}, \sum_{i \in N} \Phi_{i}(v)=\sum_{i \in N} \Phi_{i}(w)$.

Proof. First note that CTGLB can be equivalently restated like this

$$
\sum_{i \in N} \Phi_{i}(v)=\sum_{i \in N} \Phi_{i}\left(v_{S}^{*}\right)
$$

Thus it is implied by the above constant-sum requirement. To see the converse note that for any game $v$, by repeatedly applying $(.)_{S}^{*}$, that is, by repeatedly deleting minimal winning coalitions one by one, the $N$-unanimity game $u^{N}$ is finally reached. So, by repeatedly applying the former equality, one gets

$$
\sum_{i \in N} \Phi_{i}(v)=\sum_{i \in N} \Phi_{i}\left(v_{S}^{*}\right)=\ldots=\sum_{i \in N} \Phi_{i}\left(u^{N}\right) .
$$

Thus constant-sum follows immediately.

Finally, the following lemma, that gives the effect of dropping a minimal winning coalition on both power indices, will be of use in the proof of the main theorem. It shows that the effect depends on the size of the coalition and the number of players for the Shapley-Shubik index while it only depends on the number of players for the Banzhaf index.

Lemma 1 The effect on a player's power (measured by either index) of dropping one minimal winning coalition $S \neq N$ in any game $v$ is, respectively, given by

$$
\begin{gathered}
S h_{i}(v)-S h_{i}\left(v_{S}^{*}\right)= \begin{cases}\frac{(s-1) !(n-s) !}{n !} & \text { if } i \in S, \\
-\frac{s !(n-s-1) !}{n !} & \text { if } i \in N \backslash S ;\end{cases} \\
B z_{i}(v)-B z_{i}\left(v_{S}^{*}\right)= \begin{cases}\frac{1}{2^{n-1}} & \text { if } i \in S, \\
-\frac{1}{2^{n-1}} & \text { if } i \in N \backslash S .\end{cases}
\end{gathered}
$$

Proof. It easily follows from formulae (1) and (2), observing that for any $S \in M(v)$ $(S \neq N)$, the difference between $v$ and $v_{S}^{*}$ is that in $v_{S}^{*}$ any $i \in S$ is not a swinger in $S$ any more, while any $i \in N \backslash S$ becomes a swinger in $S \cup\{i\}$. 


\section{Main characterization}

Now, we have the main result in this paper: by replacing in Dubey and Shapley's systems their axioms by ours, both indices are characterized up to the choice of a zero and a unit of scale for the measure of power.

Theorem 2 Let $\Phi: S G_{n} \rightarrow R^{n}$, then

(i) $\Phi$ satisfies anonymity (An), null player* $\left(N P^{*}\right)$, symmetric gain-loss (SymGL) and constant total gain-loss balance (CTGLB) if and only if it is $\Phi=\alpha S h+\kappa \mathbf{1}$, for some $\alpha>0$ and $\kappa \in R$, where $\mathbf{1}:=(1, \ldots, 1) \in R^{n}$.

(ii) $\Phi$ satisfies anonymity (An), null player* (NP*), symmetric gain-loss (SymGL) and constant average gain-loss balance (CAGLB) if and only if it is $\Phi=\alpha B z+\kappa \mathbf{1}$, for some $\alpha>0$ and $\kappa \in R$.

Proof. (i): $(\Leftarrow)$ Let $\Phi=\alpha S h+\kappa \mathbf{1}$, with $\alpha>0$ and $\kappa \in R$. Since $S h$ satisfies An, NP* and (Lemma 1) SymGL and CTGLB, it follows easily that so does $\Phi=\alpha S h+\kappa \mathbf{1}$.

$(\Rightarrow)$ Conversely, let $\Phi$ be an index satisfying An, NP*, SymGL and CTGLB. By An, $\mathrm{NP}^{*}$ and Proposition 1, the value of the index for any null player in any game is the same. Let $\kappa$ denote this number and let $\Phi^{\prime}$ be the index $\Phi^{\prime}:=\Phi-\kappa \mathbf{1}$. It is immediate that $\Phi^{\prime}$ satisfies all the four axioms and also NP. Denote $\alpha:=\frac{\Phi_{i}^{\prime}\left(u^{N}\right)}{S h_{i}\left(u^{N}\right)}=n \Phi_{i}^{\prime}\left(u^{N}\right)$ (by An this value does not depend on $i$, and by NP and $\mathrm{NP}^{*}$ it is positive). We will prove by induction on the number of winning coalitions that for any $v \in S G_{n}, \Phi^{\prime}(v)=\alpha S h(v)$.

If $w(v)=1$, it means that $v=u^{N}$, and $\Phi^{\prime}\left(u^{N}\right)=\alpha S h\left(u^{N}\right)$ by the choice of $\alpha$.

Now assume $\Phi^{\prime}(w)=\alpha S h(w)$ for any game $w$ with a smaller number of winning coalitions than $v$. Two cases are possible.

1st case: $m(v)=1$, i.e., $v=u^{S}$ for some $S$. Then by SymGL and CTGLB,

$$
s\left(\Phi_{i}^{\prime}\left(u^{S}\right)-\Phi_{i}^{\prime}\left(\left(u^{S}\right)_{S}^{*}\right)\right)=(n-s)\left(\Phi_{j}^{\prime}\left(\left(u^{S}\right)_{S}^{*}\right)-\Phi_{j}^{\prime}\left(u^{S}\right)\right) \quad(\forall i \in S, \forall j \in N \backslash S) .
$$

Now, for any $j \in N \backslash S$, by NP, $\Phi_{j}^{\prime}\left(u^{S}\right)=0=\alpha S h_{j}\left(u^{S}\right)$, and, by the induction hypothesis, $\Phi^{\prime}\left(\left(u^{S}\right)_{S}^{*}\right)=\alpha S h\left(\left(u^{S}\right)_{S}^{*}\right)$. Then, by Lemma 1, for any $i \in S$ and $j \in N \backslash S$,

$$
\begin{aligned}
\Phi_{i}^{\prime}\left(u^{S}\right) & =\alpha S h_{i}\left(\left(u^{S}\right)_{S}^{*}\right)+\frac{n-s}{s} \alpha S h_{j}\left(\left(u^{S}\right)_{S}^{*}\right) \\
& =\alpha\left(\frac{1}{s}-\frac{(s-1) !(n-s) !}{n !}\right)+\alpha \frac{n-s}{s} \frac{s !(n-s-1) !}{n !} \\
& =\alpha \frac{1}{s}=\alpha S h_{i}\left(u^{S}\right) .
\end{aligned}
$$

So, the claim is proved for the case $m(v)=1$.

2nd case: $m(v)>1$, i. e., there are at least two different minimal winning coalitions $S$ and $T$ in game $v$. This means that by dropping first $S$, and then $T$, or the other way round, the same game is reached: 


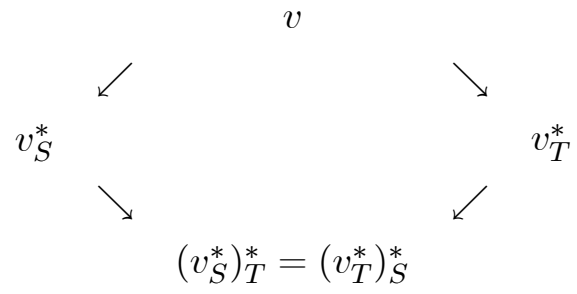

For any game $w \in S G_{n}$, any $S \in M(w)(S \neq N)$ and any $i \in N$, denote by $\Delta_{S, i}(w)$ the loss of player $i$ when the minimal winning coalition $S$ is dropped, that is

$$
\Delta_{S, i}(w):=\Phi_{i}^{\prime}(w)-\Phi_{i}^{\prime}\left(w_{S}^{*}\right)
$$

By SymGL and CTGLB it is

$$
s \Delta_{S, i}(w)=-(n-s) \Delta_{S, j}(w) \quad(\forall i \in S, \forall j \in N \backslash S) .
$$

On the other hand, by the induction hypothesis, these differences are $\alpha$-times the ones given by (3) (Lemma 1) for any game in both paths "below" $v$. This means that each time a coalition of size $r$ is dropped in either path (at least beyond the first step, in which $S$ or $T$ is dropped), the power of any player within (resp., outside) it decreases (resp., increases) in $\alpha \frac{(r-1) !(n-r) !}{n !}$ (resp., $\left.\alpha \frac{r !(n-r-1) !}{n !}\right)$. As the origin $v$ and the end $\left(v_{S}^{*}\right)_{T}^{*}=\left(v_{T}^{*}\right)_{S}^{*}$ are the same, for each player the sum of these differences must be the same for both paths. Let then $i \in S \cap T$ and $j \in T \backslash S$ (such $i$ and $j$ do exist, for the game $v$ is superadditive). Then, setting equal the effects on $i$ and on $j$, respectively, through either path we obtain:

$$
\begin{aligned}
& \Delta_{S, i}(v)+\Delta_{T, i}\left(v_{S}^{*}\right)=\Delta_{T, i}(v)+\Delta_{S, i}\left(v_{T}^{*}\right), \\
& \Delta_{S, j}(v)+\Delta_{T, j}\left(v_{S}^{*}\right)=\Delta_{T, j}(v)+\Delta_{S, j}\left(v_{T}^{*}\right) .
\end{aligned}
$$

Now, by SymGL, $\Delta_{T, i}(v)=\Delta_{T, j}(v)$ and $\Delta_{T, i}\left(v_{S}^{*}\right)=\Delta_{T, j}\left(v_{S}^{*}\right)$, then

$$
\Delta_{S, i}(v)-\Delta_{S, j}(v)=\Delta_{S, i}\left(v_{T}^{*}\right)-\Delta_{S, j}\left(v_{T}^{*}\right),
$$

and by SymGL and CTGLB, $\frac{-s}{n-s} \Delta_{S, i}(v)=\Delta_{S, j}(v)$ and $\frac{-s}{n-s} \Delta_{S, i}\left(v_{T}^{*}\right)=\Delta_{S, j}\left(v_{T}^{*}\right)$. Then, the former equation yields

$$
\Delta_{S, i}(v)+\frac{s}{n-s} \Delta_{S, i}(v)=\Delta_{S, i}\left(v_{T}^{*}\right)+\frac{s}{n-s} \Delta_{S, i}\left(v_{T}^{*}\right) .
$$

From which it follows that $\Delta_{S, i}(v)=\Delta_{S, i}\left(v_{T}^{*}\right)=\alpha \frac{(s-1) !(n-s) !}{n !}$. Therefore,

$$
\Delta_{S, k}(v)= \begin{cases}\alpha \frac{(s-1) !(n-s) !}{n !} & \text { if } k \in S, \\ -\alpha \frac{s !(n-s-1) !}{n !} & \text { if } k \in N \backslash S .\end{cases}
$$


This, together with Lemma 1 and the fact that by the induction hypothesis $\Phi^{\prime}\left(v_{S}^{*}\right)=$ $\alpha S h\left(v_{S}^{*}\right)$, entails that $\Phi^{\prime}(v)=\alpha S h(v)$, too. Thus, finally, $\Phi=\Phi^{\prime}+\kappa \mathbf{1}=\alpha S h+\kappa \mathbf{1}$.

(ii): $(\Leftarrow)$ Let $\Phi=\alpha B z+\kappa \mathbf{1}$, with $\alpha>0$ and $\kappa \in R$. Bz satisfies An and NP*. From Lemma 1 it follows immediately that it satisfies also SymGL and CAGLB. Then it is immediate that $\Phi=\alpha B z+\kappa \mathbf{1}$ satisfies also the four axioms.

$(\Rightarrow)$ The proof of the converse is very similar to that of the second part of (i), and follows the same steps. Let $\Phi$ be an index satisfying An, NP*, SymGL and CAGLB. By An, NP* and Proposition 1, the value of the index for any null player in any game is the same. Let $\kappa$ denote this number and let $\Phi^{\prime}$ be the index $\Phi^{\prime}:=\Phi-\kappa \mathbf{1}$. Then $\Phi^{\prime}$ satisfies all the four axioms and also NP. Denote $\alpha:=\frac{\Phi_{i}^{\prime}\left(u^{N}\right)}{B z_{i}\left(u^{N}\right)}=2^{n-1} \Phi_{i}^{\prime}\left(u^{N}\right)$ (by An this value does not depend on $i$, and by NP and NP* it is positive). Proceeding by induction on the number of winning coalitions we will prove that for any $v \in S G_{n}, \Phi^{\prime}(v)=\alpha B z(v)$.

If $w(v)=1$, it means that $v=u^{N}$, and $\Phi^{\prime}\left(u^{N}\right)=\alpha B z\left(u^{N}\right)$ by the choice of $\alpha$.

Now assume $\Phi^{\prime}(w)=\alpha B z(w)$ for any game $w$ with a smaller number of winning coalitions than $v$. Again we distinguish two cases.

1st case: $m(v)=1$, i. e., $v=u^{S}$ for some $S$. Then by SymGL and CAGLB,

$$
\Phi_{i}^{\prime}\left(u^{S}\right)-\Phi_{i}^{\prime}\left(\left(u^{S}\right)_{S}^{*}\right)=\Phi_{j}^{\prime}\left(\left(u^{S}\right)_{S}^{*}\right)-\Phi_{j}^{\prime}\left(u^{S}\right) \quad(\forall i \in S, \forall j \in N \backslash S) .
$$

Now, for any $j \in N \backslash S$, by NP, $\Phi_{j}^{\prime}\left(u^{S}\right)=0=\alpha B z_{j}\left(u^{S}\right)$, and, by the induction hypothesis, $\Phi^{\prime}\left(\left(u^{S}\right)_{S}^{*}\right)=\alpha B z\left(\left(u^{S}\right)_{S}^{*}\right)$. Then, by Lemma 1 , for any $i \in S$ and $j \in N \backslash S$,

$$
\begin{aligned}
\Phi_{i}^{\prime}\left(u^{S}\right) & =\alpha B z_{i}\left(\left(u^{S}\right)_{S}^{*}\right)+\alpha B z_{j}\left(\left(u^{S}\right)_{S}^{*}\right) \\
& =\alpha\left(\frac{1}{2^{s-1}}-\frac{1}{2^{n-1}}\right)+\alpha \frac{1}{2^{n-1}}=\alpha \frac{1}{2^{s-1}}=\alpha B z_{i}\left(u^{S}\right) .
\end{aligned}
$$

So, the claim is proved for the case $m(v)=1$.

2nd case: $m(v)>1$, i. e., there are at least two different minimal winning coalitions $S$ and $T$ in game $v$. This means that, as in the second part of (i), by dropping $S$ and $T$ in either order the same game is reached. Now setting $\Delta_{S, i}(w):=\Phi_{i}^{\prime}(w)-\Phi_{i}^{\prime}\left(w_{S}^{*}\right)$ as in (i). By SymGL and CAGLB it is

$$
\Delta_{S, i}(w)=-\Delta_{S, j}(w) \quad(\forall i \in S, \forall j \in N \backslash S)
$$

On the other hand, by the induction hypothesis, these differences are now $\alpha$-times the ones given by (4) (see Lemma 1) for any game in both paths "below" $v$. Thus each time a coalition of size $r$ is dropped in either path (beyond the first step, in which $S$ or $T$ is dropped), the power of any player within (resp., outside) it decreases (resp., increases) in $\alpha \frac{1}{2^{n-1}}$. As both the origin and the end are the same, for each player the sum of these differences must be the same for both paths. Let then $i \in S \cap T$ and $j \in T \backslash S$ (such $i$ and 
$j$ do exist, for the game $v$ is superadditive). Then, setting equal the effects on $i$ and on $j$, respectively, through either path we obtain:

$$
\begin{aligned}
& \Delta_{S, i}(v)+\Delta_{T, i}\left(v_{S}^{*}\right)=\Delta_{T, i}(v)+\Delta_{S, i}\left(v_{T}^{*}\right), \\
& \Delta_{S, j}(v)+\Delta_{T, j}\left(v_{S}^{*}\right)=\Delta_{T, j}(v)+\Delta_{S, j}\left(v_{T}^{*}\right) .
\end{aligned}
$$

Now, by SymGL, $\Delta_{T, i}(v)=\Delta_{T, j}(v)$ and $\Delta_{T, i}\left(v_{S}^{*}\right)=\Delta_{T, j}\left(v_{S}^{*}\right)$, then

$$
\Delta_{S, i}(v)-\Delta_{S, j}(v)=\Delta_{S, i}\left(v_{T}^{*}\right)-\Delta_{S, j}\left(v_{T}^{*}\right),
$$

and by SymGL and CAGLB, $-\Delta_{S, i}(v)=\Delta_{S, j}(v)$ and $-\Delta_{S, i}\left(v_{T}^{*}\right)=\Delta_{S, j}\left(v_{T}^{*}\right)$. From which it follows that $\Delta_{S, i}(v)=\Delta_{S, i}\left(v_{T}^{*}\right)=\alpha \frac{1}{2^{n}}$. Therefore,

$$
\Delta_{S, k}(v)= \begin{cases}\alpha \frac{1}{2^{n}} & \text { if } k \in S, \\ -\alpha \frac{1}{2^{n}} & \text { if } k \in N \backslash S .\end{cases}
$$

This, together with Lemma 1 and the fact that by the induction hypothesis $\Phi^{\prime}\left(v_{S}^{*}\right)=$ $\alpha B z\left(v_{S}^{*}\right)$, entails that $\Phi^{\prime}(v)=\alpha B z(v)$, too. Thus, finally, $\Phi=\Phi^{\prime}+\kappa \mathbf{1}=\alpha B z+\kappa \mathbf{1}$.

In view of Proposition 3, we have as an immediate corollary of the previous theorem the following one, closer to that of Dubey and Shapley, in which their axioms have been stripped of their normalizing secondary ingredients and their differentiating axioms have been substituted by ours.

Theorem 3 Let $\Phi: S G_{n} \rightarrow R^{n}$, then

(i) $\Phi$ satisfies anonymity (An), null player* $\left(N P^{*}\right)$, transfer* $\left(T^{*}\right)$ and constant total gain-loss balance (CTGLB) if and only if it is $\Phi=\alpha S h+\kappa \mathbf{1}$, for some $\alpha>0$ and $\kappa \in R$.

(ii) $\Phi$ satisfies anonymity (An), null player* $\left(N P^{*}\right)$, transfer* $\left.{ }^{*} T^{*}\right)$ and constant average gain-loss balance (CAGLB) if and only if it is $\Phi=\alpha B z+\kappa \mathbf{1}$, for some $\alpha>0$ and $\kappa \in R$.

Some comments are worth here. First, the proof of the theorem sheds some light on the crucial role that the modified game plays in our characterization. Axiomatizing an index on the finite set $S G_{n}$ requires the support of some structure, and in this case the most natural and significant structure is that of a lower semilattice with $u^{N}$ as minimal element, if $S G_{n}$ is provided with the partial order $v \leq w \Leftrightarrow W(v) \subseteq W(w)$. Then an axiomatization can be naturally grounded one way or another on some kind of inductive axioms involving this partial order. This can be done implicitly, as in Dubey's transfer, or explicitly, as in our transfer* and other axioms, using the basic step $v \rightarrow v_{S}^{*}$ (note that $v$ and $v_{S}^{*}$ are "consecutive" in the sense that $v<v_{S}^{*}$ and there is no game $w$ such that $\left.v<w<v_{S}^{*}\right)$. 
Second, observe that in the proof of Theorem 2 the superadditivity of the game has been used. This raises the mathematical question of the validity of these results in the domain of general simple games, without requiring superadditivity. The answer is positive at least for Theorem 3 (note that under $\mathrm{T}^{*}$ the case $\mathrm{m}(v)>1$ in the second part of (i) and (ii) is trivial, for this yields directly $\left.\Delta_{S, i}(v)=\Delta_{S, i}\left(v_{T}^{*}\right)\right)$. As to Theorem 2 , the question remains open.

Finally, the question of the independence of the axioms can be addressed. The following examples show the independence of any of the axioms we have used with respect to the others in either system (in brackets the uninteresting for obvious "yes" and "noes"), but that of transfer* and symmetric gain-loss with respect to anonymity, null player* and constant average gain-loss balance, still unsettled.

$\begin{array}{ccccccc}\text { Example } & \text { An } & \text { NP* } & \text { SymGL } & \text { T/T* } & \text { CTGLB } & \text { CAGLB } \\ \Phi_{i}(v)=S h_{i}(v)+\kappa_{i}\left(\kappa_{i} \neq \kappa_{j}\right) & \text { No } & \text { Yes } & \text { Yes } & \text { Yes } & \text { Yes } & (\text { No }) \\ \Phi_{i}(v)=B z_{i}(v)+\kappa_{i}\left(\kappa_{i} \neq \kappa_{j}\right) & \text { No } & \text { Yes } & \text { Yes } & \text { Yes } & (\text { No }) & \text { Yes } \\ \Phi(v)=\mathbf{0} & \text { Yes } & \text { No } & \text { Yes } & \text { Yes } & \text { Yes } & \text { Yes } \\ \Phi(v)=B z(v) & \text { Yes } & \text { Yes } & \text { Yes } & \text { Yes } & \text { No } & (\text { Yes }) \\ \Phi(v)=S h(v) & \text { Yes } & \text { Yes } & \text { Yes } & \text { Yes } & (\text { Yes }) & \text { No } \\ \Phi(v)=\frac{B z(v)}{\sum_{i \in N} B z_{i}(v)} & \text { Yes } & \text { Yes } & \text { No } & \text { No } & \text { Yes } & (\text { No }) \\ \exists \Phi(v) ? & \text { Yes } & \text { Yes } & \text { No } & \text { No } & (\text { No }) & \text { Yes }\end{array}$

\section{Normalization}

Thus, we have two families of power indices defined up to two constants on $S G_{n}$,

$$
\begin{aligned}
& S H:=\{\alpha S h+\kappa \mathbf{1}: \alpha>0, \kappa \in R\}, \\
& B Z:=\{\alpha B z+\kappa \mathbf{1}: \alpha>0, \kappa \in R\} .
\end{aligned}
$$

Any two indices in the same of either of these two families are indistinguishable through the axioms we have used so far and are equivalent in the sense that they rank identically the power of any two players in any two games. Moreover, any such a pair differs only in the zero and the unit of scale. In principle these two degrees of freedom are natural in any measure.

This allows to make a clear classification of some indices that have been proposed in the literature. The Banzhaf index $(\alpha=1$ and $\kappa=0)$, the "raw" Banzhaf $\left(\alpha=2^{n-1}\right.$ and $\left.\kappa=0\right)$ and the Rae (1969) index ( $\alpha=2^{n-1}$ and $\kappa=2^{n-1}$ ) belong to BZ, while this family does not contain the "normalized" Banzhaf index, nor the Coleman indices (Coleman (1971, 1973), 
see also Brams and Affuso (1976)). Therefore the "normalized" Banzhaf index, which is obtained by multiplying the power of a player in different games by different constants, is by no means equivalent to the Banzhaf index, it displays different properties (and paradoxes). This trivial fact does not seem to have been well understood in the literature. Similarly, Brams and Affuso (1976) do the same mistake with the Coleman indices that they consider as equivalent to the normalized Banzhaf index (see also Felsenthal and Machover (1995)).

There has been some discussion in the literature on the issue of the character absolute or relative of the power indices. It has sometimes been argued that relative indices were those which add up to 1 (see Roth (1977), Felsenthal and Machover (1995)). We try to make this point clear by subdividing the above families into smaller equivalence classes from the absolute/relative point of view, from which not any pair of indices in the same family are equivalent any more.

An index $\Phi: S G_{n} \rightarrow R^{n}$ is relative if what matters in the information summarized by $\Phi$ are the proportions $\frac{\Phi_{i}(v)}{\Phi_{j}(w)}$, for any two players and any two games. An index is absolute if what matters in the information summarized by $\Phi$ are the differences $\Phi_{i}(v)-\Phi_{j}(w)$, for any two players and any two games. Consistent with this distinction, we have the following

Definition 2 Let $\Phi, \Psi: S G_{n} \rightarrow R^{n}$, then

(i) $\Phi$ and $\Psi$ are relatively equivalent, written $\Phi \sim_{R} \Psi$, if

$$
\forall v, w \in S G_{n}, \forall i, j \in N: \frac{\Phi_{i}(v)}{\Phi_{j}(w)}=\frac{\Psi_{i}(v)}{\Psi_{j}(w)} .
$$

(ii) $\Phi$ and $\Psi$ are absolutely equivalent, written $\Phi \sim_{A} \Psi$, if

$$
\forall v, w \in S G_{n}, \forall i, j \in N: \Phi_{i}(v)-\Phi_{j}(w)=\Psi_{i}(v)-\Psi_{j}(w)
$$

To avoid the problem of dividing by zero, the relative equivalence condition should be rewritten more properly like this

$$
\forall v, w \in S G_{n}, \forall i, j \in N: \Phi_{i}(v) \Psi_{j}(w)=\Psi_{i}(v) \Phi_{j}(w)
$$

It is immediate to check that both are equivalence relations. Thus, they subdivide any family of indices into equivalence classes. The following proposition (whose easy proof we omit) states that in either family relatively equivalent indices share the "zero", but possibly not the "scale", while absolutely equivalent indices share the "scale", but possibly not the "zero". 
Proposition 5 Let $\Phi, \Psi: S G_{n} \rightarrow R^{n}$ be both in the same of any of the two families $S H$ or BZ. Then

(i) $\Phi \sim_{R} \Psi$ if and only if there exists a constant $k>0$ such that $\Phi=k \Psi$.

(ii) $\Phi \sim_{A} \Psi$ if and only if there exists a constant $k$ such that $\Phi=\Psi+k \mathbf{1}$.

Therefore each of the two indices, $S h$ or $B z$ is relatively (resp., absolutely) equivalent to any index obtained by multiplying it by a positive constant (resp., adding to it a constant). Consistently with these subdivision of the families, the following purely and properly speaking normalizing axioms, together with those that characterize the families $S H$ and $B Z$, permit to single out the classes of indices relatively equivalent and absolutely equivalent to $S h$ and to $B z$.

Zero Power (ZP): For any $i \in N, \min _{v \in S G_{n}} \Phi_{i}(v)=0$.

Unit of Power (UP): For any $i \in N, \max _{v \in S G_{n}} \Phi_{i}(v)-\min _{v \in S G_{n}} \Phi_{i}(v)=1$.

The first axiom sets the zero by stating that this is the minimal power of any player, while the second one fixes the unit of scale as the range of power of any player. Adding any of these axioms or both to the ones that characterize the families $S H$ and $B Z$, one or both degrees of freedom are eliminated. ZP sets the zero, and UP the scale. Whence the following results are straightforward:

Theorem 4 An index $\Phi: S G_{n} \rightarrow R^{n}$ satisfies anonymity (An), null player* (NP*), transfer* $\left(T^{*}\right)$ or symmetric gain-loss (SymGL), constant total gain-loss balance (CT$G L B)$, and, respectively,

(i) zero power (ZP) if and only if it is $\Phi=\alpha S h$, for some $\alpha>0$.

(ii) unit of power (UP) if and only if it is $\Phi=S h+\kappa \mathbf{1}$, for some $\kappa \in R$.

(iii) zero power (ZP) and unit of power (UP) if and only if it is $\Phi=S h$.

Theorem 5 An index $\Phi: S G_{n} \rightarrow R^{n}$ satisfies anonymity (An), null player* $\left(N P^{*}\right)$, transfer* $\left(T^{*}\right)$ or symmetric gain-loss (SymGL), constant average gain-loss balance (CAGLB), and, respectively,

(i) zero power (ZP) if and only if it is $\Phi=\alpha B z$, for some $\alpha>0$.

(ii) unit of power (UP) if and only if it is $\Phi=B z+\kappa \mathbf{1}$, for some $\kappa \in R$.

(iii) zero power (ZP) and unit of power (UP) if and only if it is $\Phi=B z$.

The comparison with Dubey and Shapley is interesting here. Their systems include indistinctly and implicitly some normalizing principles embodied in their axioms. In their systems null player, Shapley total power and also Banzhaf total power embody some 
normalizing implications that only come into effect once they combine with the other axioms. Our systems instead separate carefully any normalizing principle from the intrinsic properties of the proposed measure.

As to the absolute/relative issue, we stress the fact that from this point of view (in the unique precise sense that we can think of for this dichotomy), the situation is entirely symmetric for both indices. In other words, we see no grounds to say that $S h$ is relative while $B z$ is absolute. Both indices can be used in principle as relative or as absolute indices.

\section{$7 \quad$ Variable number of players}

So far the number of players considered has been fixed. We deal now with the specification of indices to assess the power of players in voting procedures involving any number of players. This entails comparisons of the power of different players in different processes involving possibly different numbers of players.

To deal with a variable number of players the framework needs to be adjusted. One option is assuming a universe of players as in Shapley (1953). Alternatively one can use the domain $S G:=\cup_{n \geq 1} S G_{n}$. Thus an index in this case is a collection of maps $\Phi=$ $\left\{\Phi_{n}: S G_{n} \rightarrow R^{n}: n=1,2, \ldots\right\}$. Assuming the axioms that characterize the aforementioned families for each number $n$ of players -that in what follows to avoid ambiguity we call $S H_{n}$ (resp., $B Z_{n}$ ) and similarly $S h_{n}$ (resp., $B z_{n}$ )-, it yields the family of indices given by collections $\left\{\Phi_{n}=\alpha_{n} S h_{n}+\kappa_{n} \mathbf{1}: n=1,2, \ldots\right\}$ (resp., $\left\{\Phi_{n}=\alpha_{n} B z_{n}+\kappa_{n} \mathbf{1}: n=1,2, \ldots\right\}$ ), where $\alpha_{n}$ are positive numbers and $\kappa_{n}$ arbitrary numbers. To single out an index from this family some principles must be postulated relating the power of players in games with different numbers of players. Natural references for such a comparison are what intuitively are the extreme roles: null players and dictators.

Null Players Equivalence (NPEQ): The power of any two null players in any two games, whatever their number of players, is the same.

Dictator Players Equivalence (DPEQ): The power of any two dictators in any two games, whatever their number of players, is the same.

Adding the first principle to the former systems yields that for all $m, n \geq 0, \kappa_{n}=\kappa_{m}$. Adding both equivalence principles yields in addition that for all $m, n \geq 1, \alpha_{n}=\alpha_{m}$. So, we have the natural extension of Theorems 2 and 3 .

Theorem 6 Let $\Phi=\left\{\Phi_{n}: S G_{n} \rightarrow R^{n}: n=1,2, \ldots\right\}$, then 
(i) $\Phi$ satisfies anonymity (An), null player* $\left(N P^{*}\right)$, transfer* $\left(T^{*}\right)$ or symmetric gainloss (SymGL) and constant total gain-loss balance (CTGLB) on $S G_{n}$ (for any $n \geq 1$ ), and the null players (NPEQ) and dictator players (DPEQ) equivalence principles if and only if it is, for some $\alpha>0$ and $\kappa \in R$,

$$
\Phi=\left\{\Phi_{n}=\alpha S h_{n}+\kappa \mathbf{1}: n=1,2, \ldots\right\}
$$

(ii) $\Phi$ satisfies anonymity (An), null player* $\left(N P^{*}\right)$, transfer* $\left(T^{*}\right)$ or symmetric gainloss (SymGL) and constant average gain-loss balance (CAGLB) on $S G_{n}$ (for any $n \geq 1$ ), and the null players (NPEQ) and dictator players (DPEQ) equivalence principles if and only if, for some $\alpha>0$ and $\kappa \in R$,

$$
\Phi=\left\{\Phi_{n}=\alpha B z_{n}+\kappa \mathbf{1}: n=1,2, \ldots\right\}
$$

Finally, adding similar normalizing assumptions to those used in the former subsection, both indices, that is, $S h=\left\{S h_{n}: n=1,2, \ldots\right\}$ and $B z=\left\{B z_{n}: n=1,2, \ldots\right\}$ are characterized. Note here the difference between Banzhaf and "raw" Banzhaf. The first one is singled out (up the two constants) adding the former two simple equivalence principles. But for "raw" Banzhaf no simple equivalence principle together with null players equivalence principle characterizes it.

\section{Concluding remarks}

In some sense this paper is a new turn of the screw, twenty years later, in the original motivation of Dubey and Shapley's to find a set of axioms from which to derive the Banzhaf index comparable to that obtained by Dubey (1975) for the Shapley-Shubik index:

"..up to now, the $S$-S index has attracted the lion's share of attention from game theorists, partly because of certain perceived naturalness in its mathematical foundations and partly as a by-product of research devoted to its parent solution concept, the "Shapley value" for general cooperative games. With the idea of redressing this imbalance, we have undertaken here to investigate the Bz index from a mathematical point of view." (Dubey and Shapley, 1979).

The original motivation of this paper was in fact redressing this imbalance even further. In particular, Dubey and Shapley's pair of differentiating axioms for the Shapley-Shubik index and the Banzhaf index seemed to us rather asymmetric concerning their appeal. On one hand, "efficiency", with the favorable prejudice from most game theorists. On the other, a strange axiom with some ad hoc flavor. For us it was clear from the very beginning that efficiency was arguable in the context of simple games as models of decision-making 
procedures (Laruelle and Valenciano (1999)), but balanced substitutes for efficiency and the corresponding axiom for Banzhaf were needed to show how superficial this asymmetry was.

In this search we were driven to find transparent alternatives for Dubey and Shapley's axioms, stripping them of their "normalizing" secondary ingredients. Our formulation of transfer seems more transparent than Dubey's and sheds some light on the meaning of this condition. Then transfer was replaced by a more simple and compelling assumption, symmetric gain-loss. In the case of the dichotomy "efficiency" (or, in our more neutral terms, "Shapley total power") versus "Banzhaf total power", this is achieved by the alternative dichotomy "constant total/average gain-loss balance". These axioms are remarkably close, both being a special case of a general principle satisfied by the semivalues. Moreover, both have a clear meaning and are similarly compelling. Thus both indices appear on a same footing when they are interpreted as measures of power in collective decision-making procedures. Moreover, our characterizations, separating neatly the purely normalizing conventions, contribute to a better understanding of the common mistake of considering equivalent the Banzhaf index, its "normalization", the Coleman indices and the Rae index.

It is worth stressing the crucial role that the modified game, resulting from dropping just one minimal winning coalition in a game, plays in our axiomatic systems. It arose involved in what we think is a more clear and transparent way of stating Dubey's transfer. But significantly it appears also in other axioms as "symmetric gain-loss", "constant total gain-loss balance" and "constant average gain-loss balance". While in the traditional characterization the corresponding axioms (namely "transfer", "efficiency" and "Banzhaf total power") do not share any common feature. Interestingly enough, this modified game appears also naturally if Young's (1985) coalitional monotonicity is restricted to simple games.

Finally, the results presented in this paper suggest several lines for further research. First, investigating the meaning of the axioms used here in the domain considered by us in Laruelle and Valenciano (1998), that is, the convex hull of the set of simple superadditive games, interpretable as lotteries on collective decision processes. Second, a similar critical review of the axiomatization of other semivalues as well as of other power indices proposed in the literature would be interesting. Third, it is worth studying the possibility of adapting or extending our axioms to the domain of general TU games. 


\section{References}

[1] Albizuri, J. M. and L. Ruiz, 1999, Two New Axiomatizations of the Banzhaf Semivalue, forthcoming in Spanish Economic Review.

[2] Banzhaf, J., 1965, Weighted voting doesn't work : A Mathematical Analysis, Rutgers Law Review 19, 317-343.

[3] Banzhaf, J. F., 1966, Multi-Member Electoral Districts - Do they violate the "One Man, One Vote" Principle? Yale Law Journal 75, 1309-1338.

[4] Bolger, E. M., 1982, Characterizing the Banzhaf and Shapley Values Assuming Limited Linearity, International Journal of Game Theory 11, 1-12.

[5] Brams, S. J., and P. J. Affuso, 1976, Power and Size: a New Paradox, Theory and Decision 7, 29-56.

[6] Calvo, E., and J. C. Santos, 1999, Weighted Weak Semivalues, forthcoming in International Journal of Game Theory 28.

[7] Coleman, J. S., 1971, Control of Collectivities and the Power of a Collectivity to Act. In Lieberman, B., ed.: Social Choice, 269-300, Gordon and Breach, London.

[8] Coleman, J. S., 1973, Loss of Power, American Sociological Review 38, 1-17.

[9] Deegan, J. and E.W. Packel, 1978, A New Index of Power for Simple n-Person Games, International Journal of Game Theory 7, 113-123.

[10] Dubey, P., 1975, On the Uniqueness of the Shapley Value, International Journal of Game Theory 4, 131-139.

[11] Dubey, P., and L. S. Shapley, 1979, Mathematical Properties of the Banzhaf Power Index, Mathematics of Operations Research 4, 99-131.

[12] Einy, E. 1987, Semivalues of Simple Games, Mathematics of Operations Research 12, 185-192.

[13] Felsenthal, D. S., and M. Machover, 1995, Postulates and Paradoxes of Relative Voting Power - A Critical Reappraisal, Theory and Decision 38, 195-229.

[14] Feltkamp, V., 1995, Alternative Axiomatic Characterizations of the Shapley and Banzhaf Values, International Journal of Game Theory 24, 179-186.

[15] Haller, H., 1994, Collusion Properties of Values, International Journal of Game Theory $\mathbf{2 3}, 261-281$. 
[16] Holler, M. J., and E, W. Packel, 1983, Power, Luck and the Right Index, Journal of Economics 43, 21-29.

[17] Johnston, R. J., 1978, On the measurement of Power: Some Reactions to Laver, Environment and Planning A10, 907-914.

[18] Khmelnitskaya, A., 1999, Power Indices without the Transfer Axiom. In Logic, Game theory and Social choice, Proceedings of the International Conference LGS 99, May 13-16, 1999, Harrie de Swaart ed., Tilburg University Press, Tilburg, The Netherlands.

[19] Laruelle, A., 1999, On the Choice of a Power Index, IVIE Discussion Paper WP-AD 99-10, Instituto Valenciano de Investigationes Economicas, Valencia, Spain.

[20] Laruelle, A., and F. Valenciano, 1998, On the Measurement of Inequality in the Distribution of Power in Collective Decision-making, working paper DT5/1998, Dpto. Economía Aplicada I, Universidad del País Vasco, Bilbao, Spain.

[21] Laruelle, A., and F. Valenciano, 1999, Why should Power Indices be efficient? Mimeo.

[22] Lehrer, E., 1988, An Axiomatization of the Banzhaf Value, International Journal of Game Theory 17, 89-99.

[23] Nowak, A. S., 1997, On an Axiomatization of the Banzhaf Value without the Additivity Axiom, International Journal of Game Theory 26, 137-141.

[24] Owen, G., 1978, Characterization of the Banzhaf-Coleman index, SIAM Journal of Applied Mathematics 35, 315-327.

[25] Owen, G., 1982, Game Theory, Academic Press, second edition, New York.

[26] Rae, D. W., 1969, Decision Rules and Individual Values in Constitutional Choice, American Political Science Review 63, 40-56.

[27] Roth, A., 1977, Utility Functions for Simple Games, Journal of Economic Theory 16, 481-489.

[28] Shapley, L. S., 1953, A Value for n-Person Games, Annals of Mathematical Studies 28, 307-317.

[29] Shapley, L. S., and M. Shubik, 1954, A method for Evaluating the Distribution of Power in a Committee System, American Political Science Review 48, 787-792. 
[30] Straffin, P. D., 1982, Power Indices in Politics. In S .J. Brams, W. F. Lucas and P. D. Straffin, Jr. (eds): Political and Related Models, 256-321, Springer Verlag, New York.

[31] Weber, R. J., 1979, Subjectivity in the Valuation of Games. In O. Moeschlin and D. Pallaschke (eds): Game Theory and Related Topics, 129-136, North Holland Publishing Co, Amsterdam.

[32] Weber, R. J., 1988, Probabilistic Values for Games. In A.E. Roth (ed.): The Shapley Value: Essays in Honor of Lloyd S. Shapley, 101-119, Cambridge University Press, Cambridge.

[33] Young, H. P., 1985, Monotonic Solutions of Cooperative Games, International Journal of Game Theory 14, 65-72. 